\title{
AN INDEX TO THE LITERATURE OF STEREOCHEMISTRY,
}

WITH A NOTE ON THE STEREOCHEMIBTRY OF CREATININES.

\author{
By Dr. ARnold EiloARt.
}

In compiling this index I have been much indebted to the numerous references in Van't Hoff-Meyerhoffer's "Stereochemie," most of which, with amplifications and some corrections, are incorporated here ; also to the bibliography, by Prof. Warder, of which I have made free use. To Dr. Henry Carrington Bolton my thanks are due for valuable advice as well as for bibliographical data; in particular for calling my attention to Swedenborg's contribution to stereochemistry.

\section{PLAN OF INDEX.}

The bibliography of this subject published two years ago by Warder having been classified according to subjects, I have arranged the present list in the order of the authors' names, hoping that thus the maximum benefit may accrue from the existence of both indexes, which may be used together. But in order that this index may be capable of independent use as a guide to the literature of any given branch of the subject a symbol is prefixed to each paper, excepting only those of scope too wide to admit of such brief indication of their subject matter. A list of symbols precedes the list of papers.

For clearness, only the main subject of each paper has been in- 
dicated by a symbol; thus a paper marked $\bigcirc$ may treat not only of ring-configurations but of rings containing an asymmetric carbon-atom (C) ; yet if ring-configuration is the main subject, the symbol $\mathbf{C}$ will not appear.

Whether or not a paper on optical activity bears mainly on the question of the asymmetric carbon-atom is often difficult to decide. For most cases I have settled the question by marking with Conly papers which were printed after those of Van't Hoff and Le Bel in 18\%4. In doing this I have simply accepted as a separate epoch the period which these chemists made such by their work. Earlier papers on the action of substances on polarized light are marked L for "Light."

As the great bulk of stereochemical investigation has been massed together in point of time, a chronological index seemed useless; on the other hand many a reader may wish to consult the literature of a particular period or to see at a glance the general chronological distribution of the literature; the few lines necessary to satisfy such readers have therefore been added in the form of a classification of publications into periods.

Finally, since a specially helpful form of guidance consists in information as to other guides, and since so many must make compactness and not originality the criterion of what they shall read, a list of reviews of the main subject and of its chief branches is given.

\section{LIST OF SYMBOLS AND CONTRACTIONS.}

$\mathrm{C}=$ stereochemistry of carbon.

$\mathrm{C}=$ asymmetric carbon.

$\mathrm{C}$

I for $1=$ ethane derivatives.

$\mathrm{C}$

$\|$ for $\|$ = ethylene derivatives. 


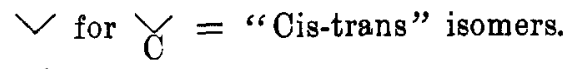

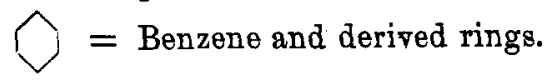

$\mathrm{L}=$ Action on polarized light.

$\mathrm{N}=$ Nitrogen except pentavalent nitrogen.

$\mathrm{N}^{\mathrm{V}}=$ Pentavalent nitrogen.

Ann. = Ann. Ohem. (Liebig.)

Ber. = Ber. d. Chem. Ges.

R. = My review of Stereochemistry. ${ }^{1}$

The figures following $R$ indicate the page or pages of the review on which the reference or references preceding $\mathrm{R}$, and placed in parenthesis with it, are given. This affords a ready means of gaining some idea of the contents of the papers so bracketed.

${ }^{1}$ About to appear independently with this index as an appendix. 


\section{LIST OF PUBLICATIONS. ${ }^{1}$}

1. I Alexander, H.-1888. Ann. 248, 281. Phenylmalic acids.

2. C Anschütz, R.-188\%. Ber. 18, 1949. The number of inactive malic acids.

188\%. Am. Chem. J. 9, 253; Chem. Ztg.

11, 1212; Ann. 239, 161.-1889. (Ann. 254, 168.-R. 20). Fumaric and maleïc acids.-1890. (Ann, 259, 145-R. 31).

66

and Bendix, P.-1890 Ann. 259, 61. Diphenylsuccinnic acids. Relationship probably same as between the hydrobenzoins.

C " and Hintze, C.-1885, Ber. 18, 1394. Attempt to "double" oxalic acid.

188\%. Ann. 239, 164. A ttempt to "double" fumaric acid.

11 " and Selden, Ch. C. -188r Am. Chem. J. 9, 3\%9; Ber. 20, 1382. Monobromeinnamic acids.

3. C Antrick, O.-188\% Ber. 20, 310. Cocaïne.

4.

II

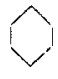

$\checkmark$
Armstrong, H. E. $-188 \%$ (J. Chem. Soc. 51, 264.-R. 14).

1888. Ibid. Proc. 54, 93 (Ref. Chem. News 58, 240). Plane and axial symmetry.

1892. Chem. News 65, 285. New structural formulæ suggested for acids supposed to be sterecmeric. ${ }^{2}$

${ }^{1}$ In several instances the references to the Berichte given in this index differ from those in the Berichte indexes, which contain numerous errors; some of them really recherches.

2 This word, introduced by Van't Hoff-Meyerhoffer, is used instead of the awkward "stereo-isomeric." 
5. II Aronstein, L., and Hollemann, A. F.-1889 Ber. 22, 481. Addition of $\mathrm{H}$ to acetylene compounds.

6. - Ashe, Isaac.-1889. Chem. News 60, 235. Forms of Atoms.

\%. - Aubin, Oh. See Graebe, C.

8. II Autenrieth, W.-188\%. Ber. 20, 1531. Thiophenylcrotonic acids. (E. Baumann's laboratory.)

9. Auwers, K.-1890. "Die Entwicklung der Stereochemie. Theoretische und experimentelle Studien." Heidelberg : C. Winter, pp. 157. 1890. Ber. 23, 399.

1891. Ber. 24, 1776. Hydrobenzoïns.

1890 and ' 91. Ber. 23, $1600 ; \mathbf{2 4 ,} 4012$.

Dimethylglutaric acids.

$\mathrm{N} \quad \because \quad$ and Dittrich, M.-1889. Ber. 29, 1996.

Structure of benziloximes.

and Jackson, Louis L.-1S90. Ber. 23,

1599. Bischoff's dynamic isomerism.

and Köbner, E.-1891. Ber. 24, 1923.

Symm. dimethylglutaric acids, etc.

and Meyer, V.-1888. Ber. 21, $\% 84,3510$.

Two benzildioximes.

1889. Ber. 22, 53\%. Two benzilmonoximes.

Ber. 22, 564. Structural identity of benzilmonoximes.-Ber. 22, 705. The third benzildioxime.-Ber. 22, 1985. No isomeric oximes of phenanthraquinone.

Ber. 22, 2011. Tetramethylsuccinic acids. Ber. 22, 3005. Dibasic acids, $\mathrm{C}_{8} \mathrm{H}_{14} \mathrm{O}_{4}$.

Naturwiss. Rundschau 4, 4\%7-481. Present state of stereo-chemical investigation. (Ref. Chem. Centrbl. [\$] 1, II., 668. 1890. Ber. 23, 101. Formation of anhydrides of acids of succinic series. Facilitated by presence of alkyls. 
$\mathrm{N}$ Auwers, K., and Meyer, V.-(Ber. 23, 2063.-R. 43.) Oximes of halogenised benzophenones. (Ber. 23, 2403.-R. 44.) Configuration of hydroxylamine.

I (Ber. 23, 20\%9.-R. 25.) Ethane derivatives. 1891. (Ber. 24, 326\%.-R.4\%.) Oximes. Answer to Claus.

Ber. 24, 4225.-R. 44. Isomeric hydrazones. $\mathrm{N} \quad$ " and Siegfeld, M.-1892. Ber. 25, $259 \%$. Benziloximes. Testing structural identity.

- Auwers, K. See Fehrlin, H. C.

\section{- " " "Krause, A.}

- " " Meyer, V.

10. - Bachmeyer, W.-1886. Chem. Centrbl. [3] 16, 209. Molecular spheres and molecular structure.

11. II Bader, R.-1890. Ztschr. phys. Chem., 6, 289 (espec. p. 315). Affinity constants.

12. $\checkmark$ Baeyer, A. von.-18\%0. Ann. Supp. 7, 1. Mellithic acid. 1885. Ber. 18, 674, (2269.-R. 10). Polyacetylene compounds. (See especially p. $22 \sim 8$. ) $\widehat{1}\}$ 1886-'91. Ber. 19, 1797; (23, 1272.-R. 10). Ann. 245, 103; 251, 257; 256, 1 ; 258, 1,$145 ; \mathbf{2 6 6}, 169$. Constitution of benzene. ${ }^{1}$ (R. 14, 26.)

1888. Ann. 246, 383. Note on L. Meyer's Benzene formula.

1892. Ann. 269, 145. Constitution of benzene.

13. N Baker, H. B. -1882. Chem. News, 48, 18i. Nitrogen and bydrogen said not to combine in presence of spongy platinum. (But see Johnson, G. S.)

14. Bamberger, E.-1890. (Ann. 257, 1; J. prakt. Chem. [2] 42, 188.-R. 14, 15.)

C

Ber. 23, 291. "Doubling" of ac. 1, 5, tetrahydronaphthalene diamine.

15. II Bandrowski, E.-18\%9. (Ber. 12, 2212.-R. 18.)

'A special connection between para-atoms (Claus' formula), said to exist side by side with the general central connection among all six atoms. 
16. - Barbieri, J. See Schulze, E.

1\%. C Baumann, E.-1882. Ber. 15, 1731. Cystine derivatives. Activity and $\mathbf{C}$ disappear together.

" See also Autenrieth, W.

$\checkmark \quad$ and Fromm, E.-1891. (Ber. 24, 1419.-R.15). 18. N Beckmann, E.-1887-'90. (Ber. 20, 2766; 22, 429, 514, 1531, 1588; 23, 1680,3319, 3331.-R. 43.) Benzaldoximes.

1889. Ann. 250, 322. Camphor series.
(Theory, pp. 360-3i5.)
See Günther, E.
see Pleissner, M.
19. - Beckurts, H. - See Otto, R.
20. N Behrend, R.-1890. (Ber. 23, 454, 17r6-R. 38, 45.)
N-poles.
and König, E.-1890. Ber. 23, 2750. Two
isomeric paranitrobenzylbenzaldoximes.
and Leuchs, K.-1889-90. Ber. 22, 384,
613; Ann. 252, 44; 257, 203. Hydroxyl-
amine derivatives.
and Nissen, Detlev.-1892. Ann. 269, 390.
O-chlorbenzaldoximes.

21. C Beilstein, F., and Wiegand, E.-1883. Ber. 17, 2261. Tiglic and angelic acids.

22. - Bendix, P. See Anschütz, R.

23. C Berthelot, D.-18r5. (Bull. Soc. chim. (Paris) [2], 23, 338.-R. 49.)-1876 and 1878. Comptes rend. 82, 441, 85, 1181 . Styrolene said to be optically active. Compare Hoff, J. H. van't, also Krakan.

24. - Besiedka, A. See Zelinsky, N.

25. I Bethmann, H. G.-1890. (Ztschr. phys. Chem. 5, 385-R. $25,28$.

26. L Biot, J. B.-181\%. Mém. de l'Académie de Paris 241 (especially pp. 114 et seq.). Turpentine vapor optically active.

1 The date is a better guide than the volume, as the series seem to be confused. 
I Biot, .T. B.-1818. Ibid 13, 144(\%). Ann. chim. phys. [?] 9, 3i2.

1819. Ann. chim. phys, 10, 63. Optical activity of rarious substances.

1835. Ibid. 13, 39. Applications to organic chemistry.

1838. Ibid. 15, 93. Optical activity of mixtures and combinations.

1838. Ibid. 69, 22. Polarized light shows difference between isomers.

1839. Comptes rend., 9, 621. Aetivity of camphor.-Ibid. 9, 825. Turpentine. (Comp. Deville.)

184t. Ann. ohim. pilys. [3] 10, 5, 1\%5, 30\%, $385: 11,8 \%$ Cse of polarized light for studying chemical mechanies.

1849-50. Comptes rend., 27, $461: \mathbf{9 9}, 433$;

31,601. Reports on Pasteur's work on the tartaric acids.-Ibid. 28, 3:21: Camphoric acid (comp. Bouchardat).-Tbid. $\mathbf{2 9 , 6 8 1 ;}$ Amu. chim. plyss. [3] 28, 215, 351. Rotation by solids.-Comptes rend., 30, r.21; Anu. chim, plyss, 99, 135, 341. Tartaric acids.

Comptes rend. 31, 101. Ann. chim. phys, $\mathbf{2 9}, 430$. Laws of rariation of rotatory porver.

185\%. Comptes rend. 33, 549. lieport on Pasteur's paper in Ann. chim. phys. [3] $34,30$.

2. । Bischoff, C. A.-1886-'8\%. Ber. 19, 95; 20, 2988. Substituted succinic acids.

1888. Ber. 21, 20\%1 (especially p. 20\%4). Nitrostilbenes.-Ber. 21, 2102. Symm. dethylsuccinic acids; especially pp. 2105 et ser.

See note on last page. 
N Bischoff, C. A.-1889. Ber. 22, 1774, 1792, 1809. Piperazines and derivatives.

1889-'90. Ber. 22, 3179; 23, 13 . Substituted succinic acids.

1890. (Ber. 23, 1924-R. 21.)

(Ber. 23, 1967-R. 47.) Ber. 23, 1972. Piperazine group.

(Ber. 23, 620, 623, 1464, 3414; 24, 1041, 1050, 1064, 10r $4,1085,2001 .-$ R. 26, 3034.) Dynamic isomerism.

1891. Ber. 24, 2083. Substituted succinic acids.

and Hausdörfer, A.-1890. Ber. 23, 1981, 198\%, 1991, 199\%, 2003. Piperazine derivatives.

66$$
1
$$

$\therefore$

66

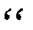

$\mathrm{N}$

I

$\mathbf{N}$

6

66

66

and Hjelt, E. - 1887 and 1888. Ber. 20, 2988, 30\%8; 21, 2089, 2097, 2102; 22, 6\%; 23, 650. Substituted succinic acids.

and Kullberg, A. von.-1890. Ber. 23, 634, 1942. Substituted succinic acids.

and Mintz, N.-1890. Ber. 23, 647, 653, 656, 3410. Substituted succinic acids.

and Nustvogel, 0.-1889. Ber. 22, 1783, 1786, 1804.

1890. Ber. 23, 2026, 2031, 2035, 2040, 2047, 2051. Piperazine derivatires.-Ber. 23,

2055, Attempts to prepare rings containing two nitrogen and two, three or six carbon atoms.

“ and Rach, C.-1885-'86. Ber. 18, 1202;

Ann. 234, 54 (especially p. 86). Symm.

dimethylsuccinic acid.

and 'Trapesunzjanz, Uh.-1890. Ber. 23,

19r\%. Diphenylpiperazine.

and Voit, E.-1889-'90. Ber. 22, 389; 23,

639, 644. Symm. dimethylsuccinic acids. and Walden, P.-1889. Ber. 22, 1812, 1819.

Disubstituted succinic acids. 
I Bischoff, C. A., and Walden, P.-1890. (Ber. 23, 1950. -R. $\%$.)

28. II Blank, A.-1888. (Ann. 248, 1.-K. 24.) Members of the stilbene group. (Communicated by Wislicenus, J, ) Compare Eiloart, A., and Redzko, IV. G.

29. II Bleibtreu, H.-1846. Ann. 59, 183. Cumaric acid.

30. C Börnstein, E., and Herzfeld, Al. -1885 . Ber. 18, 3353. Trioxybutyric acid.

31. - Boltzmann, L. -1889. Verein deutsch. Naturforscher, Sept. 21. (Ref. Chem. Centrbl. [4] 1, II, 6ii.) Relation between size of molecule and space occupied by the valences.

32. - Bosshard, E. See Schulze, E.

33. L Bouchardat, G.-1843. Ann. chim. phys. [3] 9, 213. Optical activity of alkaloids.

1844-'45. Comptes rend. 18, 298; 20, 1635. Optical activity of salicine, etc.-Ibid. 19, 601, 11\%4. Amygdaline and amygdalic acid.

1849. Comptes rend. 98, 319. Camphoric acid. Comp. Biot.

1872. Ibid 74, 665. Dulcite derivatives.

C " and Lafont.-188\%. Comptes rend. 105, 11\%\%. Borneols.

34. N Braun, E.-1889. Ber. 22, 55\%. Benzilmonoxime.

35. - Brown, F. D.-1881. Chem. News, 44, 195. Affinity and valence.

36. - Browne, G. M. See Michael, A.

37. - Bredig, G. See Will, W.

38. C Bremer, G. J. W.-1880. Ber. 13, 351. Recueil trav. Chim. 4, 180. "Doubling" of malic acid. 1885. Ibid. 6, 255. Effect of different solvents on specific rotation.

C $"$ and Hoff, J. H. van't-18r6. Ber. 9, 215. Optical activity disappears with $\mathbf{C}$. 
39. II Bruck, P.-1891. Ber. 24, 4118. Addition of iodine to unsaturated acids.

1892. Ber. 25, 503. Correction of above.

40. It Brühl, J. W.-1881. Ber. 14, 2\%42. Citraconic and mesaconic acids.

188\%. Ztschr. phys. Chem. 1, 30r; Ber. 20, 2288. Refractive power and oonstitution of benzene and naphthalene compounds.

1892. Ber. 25, 1952. Trimethylene. Straintheory.

4]. $\therefore$ Buchner, E., and Dessauer, H.-1892. Ber. 25, $114 \%$. Carboxyl derivatives of phenyltrimethylene.

42. - Buchstab, I. See Zelinsky, N.

43. $\mathrm{N}^{\top}$ Burch, G. J. and Marsh, J. E.-1889. (J. Chem. Soc., 55, 656.-R. 40.)

44. - Caberti, L. See Minunni, G.

45. - Carnelley, T.-1882. Phil. Mag. [5], 13, 112, 180. Influence of atomic arrangement on the physical properties of substances.

46. I Chalanay, L., and Knoevenagel, E.-1892. Ber. 25, 289. Stereomeric diphenylsuccinic nitriles.

47. L Chancel, G.-1869. Comptes rend. 68, 659, 726. Propyl alcohol said to be active; but see Henninger, A.

48. L Chautard, J.-Jsb. Chem. 1863, 556. The two camphoric acids.

49. II Ciamician, G.-1888. Ber. 21, 1621. Apiol and isapiol. C " and Magnanini, G.-1886. Gazz. chim.16, 390. Two tetrabromides of piperylene. 1888. Ber. 21, 1434. and Silber, P.-188\%. Ber. 20, 2594. Formation of maleïc acid from pyrrol derivatives. 50. - Clarke, F. W.-18r5. Am. Chemist, 6, 81; Proc. A. A. A. S., 24, 99. Chemistry of three dimensions. 51. - Claus, Ad.-186\%. "Theoretische Betrachtungen und deren Anwendung zur Systematik der organischen Chemie." Freiburg. 
Claus, Ad.-188\%. Ber. 15, 1405.

188\%. Ber. 20, 1422.

1888-'89. J. prakt. Chem. [2] 37, 455 (40,69;

42, 24. 260.-R. 14). Constitution of benzene

$\mathrm{N}$ 1891-92. (Ibid. [D] 44, 312; 45, 1, 37\%.-R.

4\%.) Oximes.

1892. Ibid [2] 45,556. V. Meyer and the oximes. Ibid 46, 4 i4. Oximes.

52. - Cloez, Ch. See Grimaux, E.

53. C Colson, A.-1892. Comptes rend. 114, 175. Diacetyltartaric acid.-Ibid. 114, 417. Answer to Le Bel's paper on the above, $q . v$.

53a.- Corselli, G. See Minunni, G.

54. I Cramer, C.-1891. Ber. 24, 1198. Monoximes of succinic acid.

1892. Oximidoacetic acid, etc. (Hantzsch's laboratory.)

55. - Curtiss, R. S. See Fischer, E.

56. - Debout, C. See Faworsky, Al.

5\%. C Deichmüller, A., Szymanski, F., and Tollens, B.-1885.

Ann. 298, 95. Optical activity disappears with C.

58. If Delalande, Z.-1843. Aun. 45, 334. Cumaric acid.

59. II Delisle, A.-1891. Ber. 24, 3620 .

1892. Ann. 269, 72. Transformation of unsaturated acids into their stereomers.

60. N Demuth, R., and Dittrich, M. -1891. Ber. 24, 3609. Oximes of halogenised benzophenones.

1 " and Meyer, V.-1888. Ber. 21, 264. Isodibromsuccinic acid.

61. - Dessauer, H. See Buchner, E.

62. - Deventer, Ch. M. van. See Hoff, J. H. van't.

63. I Deville,-1839. Comptes rend. 9, 824. Optical activity of chlorinated turpentine.

64. - Dittrich, M. See Auwers, K.

- " See Demuth, R.

65. N Dollfus, W.-1892. Ber. 25, 1908, 1926. Oximes. Hantzsch's laboratory. 
66. N Dunstan, W. R., and Dymond, T. S.-1892. J. Chem. Soc., 61, 470. Two acetaldoximes.

$6 \%$ - Dymond, T. S. See Dunstan, W. R.

68. C Easterfield, T. H. -1891. (J. Chem. Soc., 59, r1.-R. 6.)

69. II Ebert, G.-1882. Aun. 216, 139 ; especially p. 142.

1884. Ann. 226, 347; especially p. 353.

Coumaric acid derivatives. (Fittig's laboratory.) Compare Perkin, W. H.

70. II Eiloart, A.-1890. (Am. Chem. J., 12, 231.-R. 24.) Chlorine compounds of tolane (Wislicenus' laboratory). Compare Blank, A., and Redzko, W. G.-(J. prakt. Chem. [2] 43, 124.-R. 25.) Relative rotation of carbon atoms.

1891. Am. Chem. J., 13, 559. Solid formulæ; models.

71. C Einhorn, A.-1889. Ber. 22, 1495. Ecgonin, etc,

1890. Ber. 23, 979. Dextro-cocaine and homologues.

72. C " and Marquart, A. -1890 . Ber. 23, 468. Dextro-cocaine.

73. C Engel.-1888. Comptes rend., 106, 1734. Aspartic acid doubled.

74. II Erdmann, O.-1890. Ann. 258, 130. Benzalævulinic acids. 75. II Erlenmeyer, E.-1886. Ber, 19, 1936. Cinnamic acid series.

1890. Ber. 23, 3130. Conversion of rinnamic in to isocinnamic acid.

C “ and Hell, C.-1871. Ann. 160, 25\%. Leucine said to be inactive.

II " and Stockmeier, H.-1886. Ber. 19, 1936. Cinnamic acid derivatives.

76. C Erlenmeyer, E., Jr.-1891. Ber. 24, 2830. Phenylbromlactic acids and phenoxyacrylic acids.

77. N Ernst, W. H. See Goldschmidt, H.

78. I Evans, W. P.-1891. (Ztschr. phys. Chem., 7, 337.R. 28.) (Naumann's laboratory.) 
i9. II Faworsky, Al, and Debont, C.-1890. J. prakt. Chem. (42, 149,-R. 23.) Bromine derivatives of psendohutylene.

so. I Fehrlin, H. C.-1809. Ber. 29, 553. Bidesyls. 1890. (Ber. 93, 15it.-R, 45.) Isomeric hydrazones of o-nitrophenylglycollic acids. (Laboratory of Auwers and Meyer.)

81. ( Fischer, E.-1884, 's8 and 's9. Ber. 17, 5̃9; 21, 988, $2631 ; 0.2$, . Phenylhydrazine compounds with sugars, Pts. I, III, IV and $V$. For Pt. II, see Fischer and steche. $A$.

18ss. Verein deutsch. Naturforscher, Sept. 19th and 22d. (Ref. Chem. Centrbl. [4] 1, II. 6i2.)

1890. Ber. 23, 3\%0. Syntheses. Mammonic acid "doubled" (p. 3\%9).-Ib. 399. Synthesis of grape sugar. - Ibid 2114 . Lecture on syntheses in the sugar group, wi h bibliography. - Ibid 2611. Optical isomers of glucose, etc.-Ibid 2625. Acids of the sugar sroup.-Ibid 3684. Reduction of fruit sugar. 1891. Ber. 24, 1836, 2683. Configuration of glucose and its isomers (Ref. Am. Chem. J., 14, 399).-Ber. 24, 2136, 3622. New isomer of mueic acid,

1892. Ann. 270, 64. Sugars from glucose with more than six carbon-atoms.

C $"$ and Curtiss, R. S.--1892. Ber. 95, 1025. Gulonic acids. and Hertz, J.-1892. Ber. 95, 124i. Mucic acid.

C “ and Hirschberger, J.-1888 and '89. Ber. 21, $1805 ; \mathbf{2 9}, 365,1155,3218$. Mannose. and Meyer, J.-1889. Ber. 22, 361, 1941. Oxidation of milk-sugar and of maltose. C " and Passmore, F.-1889. Ber. 22, 359. 1890. Ber. 23, 2226. Sugar. 
C Fischer, E., and Piloty, 0.-1891. Ber. 24, 4214. The second inactive trioxyglutaric acid.

C " and Stahel, R.-1891. Ber. 24, 528. Xylose. -Ibid 539: Mannosaccharic acid.-Ibid 2144: Sorbite.

C " and Steche, A.-188\%. Ber. 20, 821. Phenylhydrazine compounds with sugars, Pt. II. C " and Tufel, J.-188\%-'89. Ber. 20, 2566, 3384; 22, 9\%. Synthetic experiments in the sugar group.

1888. Ber. 21, 1657, 21\%3. Isodulcite.

82. - Fittica, F.-1888. (Jsb. Chem., 1888, p. 8\%.-R. 49.)

83. II Fittig, R.-18ir-'84. Ann. 188, 42, 95; 195, 56; 200,

$21 ; \mathbf{2 0 6}, 1 ; \mathbf{2 0 8}, 3 \%, 111 ; \mathbf{2 1 6}, 26$.

1889-92. Ann. 255, 1, 275; 256, $50(\mathbf{2 5 9}, 1$.

一R. 23); 267, 218; 268, 1 ; 269, 1; Ber.

24, 82. Unsaturated acids.

84. N Franchimont, - - - 189\%. Versl. en Mededell Akad. Amsterdam. A second acetaldoxime. (See Junstan and Dymond.)

85. C Fock, A.-1891. Chem. Z†g., 20, r6. Crystalline form and chemical composition. (Ber. 24, 101. -R. 5.)

86. - Freer, P. See Michael, A.

8\%. Friedel, C.-1891. (Bull. Soc chim. (Paris) [3] 5, 130.R. 15.)

88. C Friedländer, P.-1890. Ber. 23, 5r2. Models. Communicated by V. Meyer.

89. X Friedländer, S.-185\%. J. prakt. Chem., 70, 449. Trimethylethylstibonium iodide said to be dextro-rotatory ${ }^{1}$ (p. 451).

90. II Friedrich, R.-1883. Ann. 219, 322, 368. Halogenised crotonic acids; especially p. 362 .

91. - Fromm, E. See Baumann, E.

${ }^{1}$ Watis' Dictionary (1863), citing Friedländer, calls the salt lævo-rotatory, and this is repeated in the edition of 1888, although Le Bel had stated in 1877 that it is inactive. 
92. I Garrett, J. C. -1888 . Ber. $21,310 \%$

93. 1 Gattermann, L.-1890. Ber. 2:3, 1333. Isomerism of certain nitrogen compounds.

$\mathrm{N} \quad$ "and Ritschke, A.--1890. Ber. 93, 1\%38.

94. - Gaudin, Marc-Antoine.-18i3. "LArchitecture du Monde des Atomes," Paris, 18:3. Gauthier-Villars, pp. xix + 231 . Compare West.

95. C Gernez, D.-1864. Ann. scient. de l'Ecole normale sup. 1, 1. Optical activity of turpentine and camphor vapors.

96. - Giesel, F. See Liebermann, C.

9\%. N Goldschmidt,-H.-1889 and '90. (Ber. 22, 3101; 23, 2163, 2r46.-R. 43.) 23, 3113. Benzaldoximes are structurally identical.

98. N “ and Ernst, H. W.-1890. Ber. 23, 21\%0. Oximes.

99. $\mathrm{N}$ “ and Kisser.-188\%. Ber. 90, 20r1. Carvoximes.

N $\quad$ and Kjellin, C.-1891. Ber. 24, 254\%. P-nitrobenzaldoximes.

100. C Goldschmiedt, G.-1888. Sitz. Ber. Akad. Wien, January ; Monatsh. Chem., 9, 42. (Ref. Ber. 240. Papaverine.)

101. I Graebe, C.-188\%. Ber. 90, 348. Diphenic acids. Formulæ.

1890. (Ber. 23, 1344.-R. 26.) Benzilorthocarboxylic acid (especially p. 134r).

and Aubin, Ch.-188\%. Ber. 20, 845, 848. Diphenic acids.

and Juillard, P.-188\%, Ann. 242, 214. Diphthalic acids.

1888. Ber. 21, 2003. Benzilorthocarboxylic acid.

102. C Grimaux, E., and Cloez, Ch.-188^. Comptes rend. 104, 1446. Erythrene bromides.

103. C Griner.-1891. Dissertation, Paris: Allylpropenyltetrabromide, etc. Chains of active carbons united to bromine. 
104. C Günther, A., and Tollens, B.-1890. Ber. 23, 2585. Fucuse, a sugar isomeric with rhamnose.

105. N Günther, E.-1889. Ann. 25:, 44. Benziloximes, etc. (Communicated by Beckmann.)

106. C Guye, P. A.-1890. Comptes rend: 110, r14. Chemical constitution and optical activity.-(Bull. Soc. chim. (Paris) [3] 3, 595.-R. 5.)

1891. "Étude sur la Dissymétrie moléculaire."

1892. Ann. Chim. phys. [6] 25, 145. Effect on optical activity of the position of the centre of gravity of the molecule relative to the planes of symmetry of the active carbon.

10\%. C Haller, A.-1884. Bull. Soc. chim. (Paris) [2] 41, 32\%.

188\%. Comptes rend., 104, 66. Derivatives of the two camphors. - Ibid 105, 22i; 109, 187. Borneols.

108. N Hantzsch, A.-1890. Ber. 23, 2322. Asymmetric monoximes.

1890 and 1891. Ber. 23, 2325, 2332; 24, 13, $31,36,51,495$. Oximes.-Ibid. 1192, 4018. Fatty oximes.-Ibid. 3479 : Nomenclature. (R. 41-4\%)

1892. Ber. 25, \%05. Oximes.-Ibid. 1692. Answer to Claus.-Ibid. 1904. Aldoxime acetic acid.-Ibid. 2164. Constitution, configuration and chemical behavior of the oximes.

N $\quad$ and Kraft, F.—1891 (Ber. 24, 3511.-R. 45). Isomeric hydrazones.

N $\quad \because \quad$ and Miolati, A.-1892. Ztschr. phys. Chem. 10, 1. Relations between configurations and affinity-constants of stereomeric nitrogen compounds.

$\mathrm{N} \quad " \quad$ and Werner, A.-1890. (Ber. 23, 11, 1243, 2764, 2769, 2773, 2776.-R. 41-4i.) 
109. C Hartmann, W.-18s8. Ber. 21, 221. Camphoric acid and salts. Relations between specific rotatory power and composition.

109a.- Haseloff, E. See Roserld.

110. - Hausdörfer, A. See Bischoff, C. A.

111. N Hausmann, J.-1890. Ber. '23, 531. Dioximes of nitrobenzil.

112. I Hell, C.-1889. Ber. *2, 6r. Symm. diethylsuccinic acids. and Mayer, W.-1889. Ber. 92, 56. Diisopropylsuceinic acids.

113. C Henninger, A.-188\%. In " "Dix années dans l'Histoire d'une Théorie." Private communication. Propyl alcohol is inactive. (Compare Chancel.)-Compt. rend., 104, 1446. Bull. Soc. chim. (Puris) [2] 48,31. Bromides of erythrite.

114. - Herman, B. See Willgerodt, C.

115. A Herrmann, F.-1888. (Ber. 21, 1949.-R. 13.)-Correction: Ibid. 2338 .

1890. Ber. P3, 2060.

“ See Hoff, J. H.van't.

116. C Herrmann, P., and Tollens, B.--1885. Ber. 18, 1333, 2608. Saccharin.

11\%. - Hertz, J. See Fischer, E.

118. C Herzfeld, A.--1883. Amn. 900, 335. Gluconic acids. “ See Börnstein.

119. C Hesekiel, A.-1885. Ber, 18, 3091. B-picoline said to be optically active. (Comp. Landolt.)

120. C Hesse, O.-18ij. Ann. 176, 89, 189. Tarious substances and polarized light.

121. II Hill, H. B. -18so. Ber. 13, ‘3t. Maleïc acid derivatives.

122. - Hinrichs, G.-18i3. Comptes rend., 76, 1592. Joments of inertia of molecules.

1891. Ztschr. phys. Chem. 8, 6r4. Mechanical determination of the linkage of carbon atoms. 
- Hinrichs, G.-1892. Comptes rend., 114, 1064. Moments of inertia.

123. - Hinsberg, O.-1890. Ann. 260, 40. Observations on selenium.

124. - Hintze, C. See Anschütz, R.

125. I Hjelt, E.-1889 and 1892. Ber. 22, 2906; 25, 488. Substituted succinic acids.

" See Bischoff, C. A.

126. C Hoff, H. J. van't.-1885. Maandblad voor Natuur wetensch. 15, 1; Ber. 18, 2170, 2713. Malic acid; there is no inactive acid which cannut be doubled. (Compare Pasteur.)

12\%. C Hoff, J. H. van't.-18\%4. "Voorstet tot uitbreidung der II tegenwoordig in de scheikunde gebruikte structuur-formules in de ruimte; benevens een daarmeê samenhangende opmerkung ourtrent het verband tusschen optisch actief vermogen en chemische constitutic van organische verbindungen." Utrecht, 8vo. ${ }^{1}$

“ 1875. Bull. Soc. chim. (Paris) [2] 23, 295. A republication of the theories given in the Dutch pamphlet.

“ 18\%5. ("La Chimie dans l'Espace." Rotter. dam: Bazendjik, pp. 44, 12mo, 2 plates.R. 15).

“ 1876. Maandblad voor Natuurwetensch, 6, 72. Styrolene is inactive. (See Berthelot, also Krakau.)-Ibid. 6, 150. Rings.

18\%. "Die Lagerung der Atome im Raume." Nach des Verfassers Broschüre "La chimie dans l'espace" deutsch bearbeitet von F. Herrmann. Nebst einem Vorwort von J. Wislicenus. Braunschweig: 8vo. ill.

1 This paper is of historic importance as being Van't Hoff's first publication on the subject. See R., p. 4. 
C Hoff, J. H., van't.-18гi-r9. Ber. 10, 1620; 11, 42;

12, 4it. Activity disappears with $\mathrm{C}$.

$\mathrm{N}$ 18r8-81.--"Ansichten über die organische

Chemie." Bramschweig. 3 parts, 8vo. (Especially pp. :9-81.-R. 3i.)

II 1884. "Etudes de dynamique chimique." Amsterdam: Pp. irtol4, 8vo. (p. 9r, fumaric and maleje acids; pp. 100, 103, mechanism of addition to unsaturated bodies).

1886. Archives Neêrl. Kon. Svenska.

Akad. Handl. Proportions in which two active isomers must be present to give an inactive product on reeiprocal conversion. 188r. "Dix années dans l'histoire d'une théorie (Deuxième édition de La chimie dans l'espace)." Rotterdam : Bazendjik, pp. 102, $12 \mathrm{mo}$.

1891. ("Chemistry in Space." Translated and edited by J. E. Marsh. Oxford, Clarendon Press. Pp. vi.t-128, 12mo.--R. 15.) 189\%. "Stereochemie. Nach ran't Hoff's

'Dix années dans l'histoire d'une théorie' neu bearbeitet von W. Meyerhoffer." Wien. Pp. viii-12r, 8vo, ill.

C $\because$ and Deventer, Ch. M. van.-1886 and 188\%. Ber. 19, 2142; Ztschr. phys. Chem. 1, 165. Influence of temperature on the doubling of racemates.

188\%. Recueil trav. chim. 6, 36, 91, $13 \%$.

Relation between "doubling" and fusion. "Point of transition."

See Bremer, G. J. W.

128. N Hoffmann, E.--1890. Ber. 23, 2064. Dioximes of cuminil. (V. Meyer's laboratory.)

1891. Ann. 264, 160. Oximes of halo genised benzophenones. 
129. C Hofmann, A. W. von.-1885. Ber, 18, 5. Coniine.

130. - Hollemann, A. F. See Aronstein, L.

131. II Holt, A.-1891 and 1892. Ber. 24, 4120; 25, 961. Erucic and brassic acids.

132. If Hölz, 0.-1889. Ann. 250, æ30. Brominated derivatives of pseudobutylene. (Wislicenus' laboratory.)

133. - Hugo, L.-1884. Comptes reud., 98, 1596. Forms of atoms.

134. - Hundt, Chr. See Ladenburg, A.

135. L Iodin, F. V.-1864. Bull. Soc. chim. (Paris) [2] 1, 432. Influence of inactive substances on optical activity of sugars.

136. - Jackson, Louis L. See Auwers, K.

137. N Janorski, J. V.-1889. Ber. 22, 1172. Monatsh. Chem. 9, $828 ; 10,585$ (Ref. Ber. 21, 795; 2:, 752). Azo- and azoxytoluenes.

$\mathrm{N} \quad \because \quad$ and Reimann, K.-1889. Ber, 22, 40. Parazoxytoluenes.

138. N Johuson, G. Stillingfleet.-1883. (J. Chem. Soc. 39, 128, 130.-R. 40.) Synthesis of ammonia.Proc. Roy. Soc. 33, 32. Allotropic nitrogen (only the title of the paper appears). -Chem. News 48, 202. Answer to H. B. Baker (q.v.).-Chem. News 48, 253, 264. Electro-chemical researches on nitrogen. 1885. (On certain indications of the exist. ence of an allotropic form of nitrogen and on the synthesis of ammonia. London: J. and A. Churchill, pp. 32, 8vo, ill.-R. 40.) (Ref. Chem. News 52, 34.)

1886. Chem. News 54, 22. Reply to Williams and Ramsay, $q . v$. 1888, '91 and '92. Proc. Roy. Soc. 43, 493; 49, 538; 50, 28\%. Creatinines. ${ }^{1}$

139. - Juillard, P. See Graebe, C.

\footnotetext{
1 See note at end.
} 
140. C Jungfieisch, E.-1Sra. Comptes rend. 75, 439, 1739. Racemic formed from tartaric acid by heat. 18.3. Bull. Soc. chim. (Paris) [2] 19, 290, 530. Camphoric acid. 18:8. Ibid 30, 14r. Bimalate of ammonium. 1880. Compt. rend., 90, 790. Camphoris acids.

1884. Bull. Scc. chim. (Paris) 41, 222, 226. "Doubling" and synthesis of optically active substances.

141. C Just, F.-1883. Ann. 220, 146. Ethanes derived from active amyl alcohol and containing $\mathrm{C}$. (Wislicenus' laboratory.)

142. - Kehrmann, F.-1890. Ber. 23, 130. (J. prakt. Chem. [2] 42, 134. Influence of space on chemical reactions. - R. 45.)

N 1892. Ibid.46,53. Dinitroso-azobenzene; a reply to Willgerodt $(q \cdot v$.$) .$

$\mathrm{N} \quad$ "6 and Messinger, J.-1892. (Ber. 25, 598.-R. 39.)

143. II Kanonnikow, J.-1883. Zhurnal Khim. I, 434. (Ref. Ber. 16, 304\%.)

144. II Kauder, E.-1885. J. prakt. Chem. [2] 31, 1. Chlorfumaric formed from dextro-tartaric acid.

145. C Keiser, E. H.-1889-'90. Am. Chem. J. 11, 2ir ; 12, $35 \%$ Sugar group. Review.-Ibid. 11, 480 . Constitution of hydroxylamine. Review.

II 1890. Ibid. 12, 99. Synthesis of fumaric acia.

146. Kekulé, A.-1865-'66. Bull. Soc. chim. (Paris). [2] 3, 98 ; Ann. 137, 129. Benzene.

II " and Strecker, 0.-1884. Ann, 293, 170. Maleïc acid from benzene.

147. - Kernbaum, A. See Walden, P.

148. C Kiliani, H.-1886. Ber. 19, 3029. Arabinose. 188\%. Ber. 20, 339. Saccharic and gluconic acids.-Ibid. 1233, Arabinite. 
C Killani, H., and Scheibler, C.-1888. Ber, 21, 3276. Sorbinose.

149. - Kisser, E. See Goldschmidt, H.

150. - Kjellin, C. See Goldschmidt, H.

151. I Klinger, H., and Standke, O.-1891. Ber. 24, 1264. "Isobenzil" is dibenzoate of tolallyl

$$
\begin{gathered}
\mathrm{C}_{6} \mathrm{H}_{5} \mathrm{COCOC}_{6} \mathrm{H}_{5} \\
\mathrm{C}_{6} \mathrm{H}_{5} \mathrm{COCOC}_{6} \mathrm{H}_{5}
\end{gathered}
$$

$\mathrm{N} \quad$ " and Zuurdeeg, J.-1889. Ann. 255, 310. Azo and azoxybenzene derivatives. Compare Janovsky.

152. I Knoevenayel, E.-1888. Ber. 21, 1359. Bidesyls. “ See Chalanay, L.

153. - Köbner, E. See Auwers, K.

154. König,Arn. - 1889. ("Zur 'Theorie und Geschichte der fünfgliedrigen Kohlenstoffringe enthal. tend eine Berechnung des Abstandes von Kohlenstoffatomen bei doppelter und einfacher Bindung sowie einen Beitrag zur Kenntnis der Hydrindenderivate." Leipzig: Lorentz, pp. 80, 8vo, ill. [Wislicenus' laboratory.]-R. 11.)

1890. Chem. Ztg. 14, 34\%. Distance between carbon atoms singly and doubly linked.

155. - König, E. See Behrend, R.

156, C Körner, W., and Menozzi, A.-188\%. Gazz. chim. 17, 226. (Ref. Ber. 21, 86.) Asparagine "doubled."

15\%. - Kolbe, H.-18\% and 's1. (J. prakt. Chem. [2] 15, $4 \% 3 ; \mathbf{2 3}, 489 .-$ R. 49.)

158. N Kraft, Fr.-1890. (Ber. :3, 2\%80.-R. 45.)

159. C Krakau, - -1878. Ber. 11, 1259. Optical activity of styrolene. Compare Berthelot, D., also Hoff. J. H. van't.

160. - Krapivin, S. See Zelinsky, N.

161. N Krause, Albelt.-1890. (Ber. 23, 361\%. The isomeric hydrazones of orthonitrophenylglycollic acid. -R. 45.) Jaboratory of Auwers and Meyer. 
162. L Krecke, ---18:1. Arch. neêrl. 6, 35t. Relation between optical activity and chemical composition. (Ref. J. prakt. Chem. [2] 5, 6.)

163. II Krusemark, P.-1880. Ann. 906, 16. Brommethylacrylic acids. (Fittig's laboratory.)

164. C Külz, E.-1882. Ber. 15, 1401. Cystine.

188t. Ztschr. f. Biologie $\mathbf{2 0}, 165$. (Ref.

Ber. 17, 534.) Oxybutyric acid.

165. - Kuhlberg, A. von. See Bischoff, C. A.

166. $\bigcirc$ Ladenburg, A.-1869. Ber. 2, 140, 1i2. Rings. 1876. "Theorie der" aromatischen Verbindungen." Braunschweig.

$\mathrm{N}^{\mathrm{v}}$

C

C

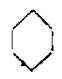

C

Q

C

$\mathrm{N}^{\mathrm{v}}$ 18\%. (Ber. 10, 561, 1152, 1634.-R. 38.) 1880. Ber. 13, 2329. Reversal of rotation. 1886. Ber. 19, 25\%8. Coniine.-Ibid. 2584, 29\%5. Piperidine bases.

1886-'8\%. Ber. 19, 9:1; 20, 6\%. Benzent. 1888. Ber. 21, 3065. Atropine.

1888-90. (Ann. 246, 382; Ber. 23, 100\%, 2688.-R. 14.) Benzene.

and Hundt, Chr.-1889. Ber. ••?, 2590, Tropic acid and atropine.

16\% C Lafont, J. See Bouchardat, G.

168. L Landolt, H.-18\%3. Ber. 6, 1282. Reply to Oudemanus $(q \cdot v$.$) .$

C

18ii. Ann. 189, 241. On optical rotatory power. Historical and critical.

18:9. "Das optische Drehungsvermögen organischer Substanzen und die praktische Anwendung desselben." Braunschweig. Pp. xii + 23r (especially p. 23).

1882. "Handbook to the polariscope." Translation of the above by Robb and Veley. London. 8vo.-R. 7.)

1880. Ber. 13, 2329. Reversal of rotation. 
C Landolt, H.-1886. Ber. 19, 15\% $\beta$-picoline is inactive. (See Hesekiel.)

169. If Langbein.-188\%. Ber. 20, 1010. A second $\alpha$-bromcrotonic acid. Communicated by Lippmann, E. O. von.

1\%0. - Le Bel, J. A.-18\%4. Bull. Soc. chim. (Paris) [2] 22, $33 \%$-R. 4 .

C 1876. Ibid. 25, 545. Derivatives of active amyl alcohol. (Compare Just, F.)

18\%. Ibid. 27, 444. Trimethylethylstibonium iodide is inactive. (Compare Friedländer, S.)

18;8-;9. Comptes rend. 87, 213; 89, $312 ; 92,532$. "Doubling" by organisms. 18\%9. Bull. Soc. chim. (Paris) 31, 104. Primary amyl alcohol.

1880. Ibid. 33, 106. Secondary amyl alcohol and iodide.-Ibid. 34, 129. Propylene glycol.

1882. Ibid. (37, 300.-R. 34) 38, 98. Orthotoluidine could not be doubled.

1889. (Comptes rend. 110, 144. Substituted ammonium chlorides.-R. 39.) Bull. Soc. chim. (Paris) [3] 2, 305 . Inactivity of methylethylpropylammonium chloride.

1890. Bull. Soc. chim. (Paris) [3] 3, 244; 4, 104. Substituted ammonium salts. (Ibid. 3, 788.-R. 3.) (Ibid. 4, 613.R. 38.)

1891. Comptes rend. 112, 724. Methylethylpropylisobutylammoniurn chloride "doubled."

1892. Comptes rend. 114, 175. Emphasizes the difference between his views and those of Van't Hoff.-Ibid. 304. On the paper of Colson $(q \cdot v$.$) .$ 
171. - Lecco, M. See Meyer, Y.

172. - Leuchs, K. See Behrend, R.

1.3. I Leuckart, R.-1885. Ber. 18, :344. Dimethylsuccinic acid; (especially p. 2348).

1r4. C Lewkowitsch, J. -158 ? and 83 . Ber. 15, 1505; 16, 1565, 1568, 9.21. Mandelic acid.-16, 2720. Glyceric and lactic acids.

1888. J. Chem. Soc., 53, 781. Optical activity of benzene derivatives.

175. I Liebermann, C. - 1890. Ber. 2:3, 141, 512. Isocinnamic acid.-(Ibid. $8510 .-$ R. 9.)

1891. Ber. 24, 1101. Allocinnamic differs only in erystalline form from isocinnamic. 1892. Ber. 95, 90. Cinnamic acids.

II “ and Sachse, H.--1891. Ber. :4, 4112. Addition of iodine to unsaturated acids.

C " and Giesel, F.-1s90. Ber. 23, 508. Cocaine.

176. C Linoissier, G.-1891. Bull. Soc. chim. (Paris) [3] 6, 10. Doubling of lactic acid. (Compare Schardinger, F.)

17\%. - Lippmann, E. O. von. See Langbein.

178. Loschmidt, J.-1890. Wiener Sitzungsber., p. 99. (Mnnatsh. Chem., 11, 28.-R. 14.)

179. N Lossen, W.-18\%-ว-92. Ann. 171, 271; 175, 25\%; 178, $213 ; \mathbf{1 8 1}, 384 ; \mathbf{1 8 2}, 214 ; \mathbf{1 8 6}, 1 ; \mathbf{2 0 5}$, $2.4 ; \mathbf{2 1 7}, 1 ; \mathbf{2 5 2}, 1 \% 0$. Ber. 24, 4059; (25, 433.-K. 45). Hydroxylamine derivatives.

1880. Ann. $\mathbf{2 0 4}$, 365. Distribution of atoms in the molecule. Compare Kolbe. 188:: Ber. 20, 3306. Arrangement of atoms in space. See Wislicenus.

180. - Lucas, F.-1868. Comptes rend. 67, 990, 1025; 68, 1313. Atomic mechanics. Mathematical.

181. - Magnanini, G. See Ciamician, G.

182. - Maisch, C. C. See Michael, A. 
183. Il Mangold, K.-1892. Monatsh. Chem. 13, 326 (Ref. Chem. Centrbl. [4] 4, II, 155.) Stereochemistry of trioxystearic acids.

184. Mann, L.-1883. "Die Atomgestalt der chemischen Grundstoffe." Berlin.

1884. "Der Atomaufbau in den chemischen Verbindungen und sein Einfluss auf die Erscheinungen." Berlin. F. Heinicke. (Ref. Chem. Centrbl. [3] 17, r86.)

185. C Maquenne,-1888. Comptes rend. 106, 286. Galactosecarboxylic acid.

186. - Marcano, V. See Muntz, A.

18\% - Marquart, A. See Einhorn, A.

188. Marsh, J. E.-1888. (Phil. Mag. 162, 426.-R. 14.)

1889. Chem. News, 60, 30\%. Camphoric acid.

See Burch, G. J.

See Hoff, J. H. van't. "Chemistry in Space" contains an appendix by Marsh.

189. I Massol, G. - 1892. Comptes rend. 114, 1373. Thermochemical investigation of dibasic acids: the distance between their carboxyl groups not altered by substituting methyl for hydrogen.

190. Matthews, F, E.-1891 and 1892. Chem. Soc. J., 599, $165 ; 61,103$. The benzene hexachlorides. Compare Frieded, $\mathrm{C}$.

191. C Jauthner, J.-1883. Sitzber. Akad. Wissensch. Wien, [2] 8.5, 882. Cystine, tyrosine.-Ztschr. physiol. Chem. 7, 222. Leucine.

192. - Mayer, W. See Hell, C.

193. II Melikoff, P., and Petrenko-Kritschenko, P.-1891. Ann. 266, 358. Crotonic and iso-crotonic acid are structurally identical.

194. - Menozzi, A.-See Körner, W.

195. - Messinger, J. See Kehrmann, F. 
196. C Meunier, J.-1890. Comptes rend. 111, 49. Mannite and surbite.

19\% - Meyer, J. See Fischer, E.

198. Meyer, Lothar--1888. Ann. 247, 251. Benzene.

199. Meyer, R.-1882. Ber. 15, 1823. Octahedral formula.

200. Meyer, Victor.-18\%. Ann. 180, 192. Union of carbon atoms.

$\mathrm{N}^{\mathrm{N}} \quad$ 18\% (Ber. 10, 309, 964, 978, 1291.-R. 39.)

- 1888. Ber. 21, 265. Form of atoms.

1890. (Ber. 23, 56\%.-R. ז.)

$\mathrm{N}$ 1891. (Ann. 264, 16.-R. 43.)

$N \quad$ “ and Auwers, K.-1889. Ber. 22, 1985.

Oximes of phenanthraquinone.

$\mathrm{N}^{\mathrm{v}} \quad \because \quad$ and Lecco, M.-18r4-' $\% 5$. Ber. 7,$1747 ; 8$, 233, r69, 936. (Ann. 180, 1;3.-R. 38.)

Substituted ammonium compounds.

| “ and Riecke, E.-1888. (Ber. 21, 946, 1620. -R. 11.)

$\mathrm{N} \quad$ and Warrington, A.-188\%, Ber. 20, 500 . Acetoximes.

$\because \quad$ See Auwers, K.

201. II Michael, Arthur.-1886. Ber. 19, 1372, 1377, 1381.

Unsaturated isomers.

188\%. Am. Chem. J. 9, 180. Alloisomerism.-Ibid. 9, 364. Levulinic and maleï acids.-J. prakt. Chem. [2] 35, 35\%.

1888-'89. (J. prikt. Chem. 38, 21; 40, 29. -R. 20, 23.) 40, 171.

1892. Ibid. 46, 209. Addition of bromine to acetylene dicarboxylic acid. Compare Bandrowski, E., also Wislicenus, J.-Ibid. 46, 266, 400,-424. Crotonic acids. General theory.

II " a and Browne, G. M.-1886. Ber. 19, 1378. Two $\beta$-isobromerotonic acids. 
I Michael, A., and Browne, G. M.-188\%. (Ber. 20, 550. R. 9.) Cinnamic series.-Am. Chem. J., 9, 274. Alloisomerism.-J. prakt. Chem. [2] 35, 25r; 36, 1r4. Crotonic acid series; $\alpha$-bromcrotonic acid. See Langbein.

11 " and Freer, P.-18s9. J. prakt. Chem. [2] 40, 95. Addition of hydriodic acid to the crotonic acids.

and Maisch, C. C.-1892. J. prakt. Chem. 46, 233. Action of sodium ethylate on dibromsuccinic ether.

11 " and Palmer, G. M. Ber. 19, 13\% ; Am. Chem. J., 9, 19r.

I) " and Pendleton, H.-1888. J. prakt. Chem. [2] 38, 1. Crotonic acid series.

" 1889. Ibid. 40, 63. Cinnamic acid series.

11 " and Schulthess, 0.-1892. Ibid. 46, 236.

Crotonic acid derivatives.

and Tissot, G.-1892. Ibid. 46, 285, 384. Homologues of malic acid.

C “ and Wing, J. F.-1884 and '85. Ber. 17, 2984; Am. Chem. J., 7, 278. Inactive aspartic acid.

202. II Michael, Helen A.-1892. J. prakt. Chem. [2] 46, 2 22. Crotonic acid.

203. Miller, A. K.-188\%. J. Chem. Soc.51,208. Review of the benzene theories of Baeyer and Thomsen.

204. C Miller, W. von.-1878. Ber. 11, 1450. Active impurities in styrolene. See Berthelot. N $\quad$ and Plöchl, J.-1892. Ber. 25, 2020. Schiff's bases. 'Three stereomeric forms of an oxime theoretically possible. and Rohde, G.-1892. Ber. 25, 201\%. $\alpha$ phenylhydrocinnamic acid; three modifications.

205. C Minkowski, 0.-1884. Centrbl. med. Wissensch., p. 242 (Ref. Ber. 17, 334). Oxybutyric acid. 
206. - Mintz, N. See Bischoff, C. A.

207. N Minunni, G. -1890 and 1891. Gazz. chim. ital. 20, $6 \pi$ (Ref. Ber, 24, 152); 21, 113 (Ref. Ber. 24, 561): 21, II, 192 (Ref. Ber. 24, 833). Oximes.

1892. Ibid. 2:, II, $1 \% 4,101,21 \%, 830$ (Ref. Chem. Centrbl. [4] 4, II, 905, 906, (10), 910.) Oximes, etc.

$\mathrm{N} \quad$ “ and Caberti, L.-1891. Gazz. chim. ital. $\mathbf{2 1}$, 136 (Ref. Ber. 24, 562). Benzaldoximes. $\mathrm{N} \quad \because \quad$ and Corselli, G.-1892. Gazz. chim. ital.

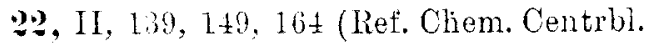
[4] t, II, 903,904$)$. Oximes.

$\mathrm{N} \quad "$ and Ortolera, G.-1892. Gazz. chim. ital. 9., II, 18:3 (Ref: Chem. Centrbl. [t] t, II, 906.) Benziloximes.

208. - Miolati, A. See Hamtzsch, A.

209. I Mulder, E.-1s68. Ztschr. Chem. 11, 58.

18\%. Ber. $;, 1329$. Relation between composition and optical activity.

210. C Mourgnes, T.-1s!uo. Comptes rend. 111, 112. Activity of hexachlorlydrin of mannite.

211. C Muntz, A., anl Narcano, Y.-1884. Comptes rend. 99, 38 . P'erseite.

212. - Nïgeli, E. Sec Schulze, l.

213. - Nastrogel, O. See Bischoti, ( $\mathrm{A}$.

214. - Naumann, Alex-1890. (Ber. 93, 4i..-h. 10.) Chem.

Ztg. 14, 219. liclative force of single, double and triple bonds. See also Erins, W. P. 215. N Tef,J, L.-186?. Ann. :30, :60: especially p.32: et seq. Benziloximes and benzaldoximes; structural differences between alleged stereomers.

216. - Nissen, Detlev. See Behrend, R.

21\% N Noyes, W. A.-1889. An. Chem. J. 11, 4!16. On Baeyer's strain theory.

218. N Nussberger, (i.-189\%. Ber. 95, 2142. Dioximes. (Hantasch's laboratory.) 
219. ' Oddo, G.-1891. Gazz. chim. ital. 21, II, 559 (Ref. Ber. 25, 329). Stereochemistry of the camphor group.

220. I Ossipoff, J.-1889. Comptes rend. 109, 223. Diphenylsuccinic acids.

221. II Ostwald, W.-1889. Ztschl. phys. Chem. 3, 170, 369. Constants of electric conductivity.

1892. (Ibid. 9, 220.-R. 49.)

222. I Otto, R., and Beckurts, H.-1885. Ber. 18, 825. Two sym. dimethylsuccinic acids. Especially p. 846.

223. L Oudemanns,. A. C., Jr.-18r3. Pogg. Ann. 148, 33\%. Influence of inactive solvents on optical activity.-Ber. 6, 1166, 1447. On the paper of Krecke $(q, v$.$) .$

C 1885. Versl. Akad. Wetensch. Amsterdam, 3, 408. In aqueous solution all tartrates have same coefficient of rotation.

223a. - Palmer, G. M. See Michael, A.

224. - Passmore, J. See Fischer, E.

225. L Pasteur, L.-1848-'58. Ann. Chim. phys. [3] 24, 442; 28, $56 ; 31,67 ; 38,437$; Comptes rend. 28, 47\%; 31, 480; 35, 176; 37, 110, 162; 46, 615 . Relations between composition, optical activity and crystalline form. Tartrates.

L'Institute, 1850, 339.

1852. Ann. Chim. phys. [3] 34, 30 (especially p. 46). Existence of an inactive malic acid which could not be doubled. Compare Hoff, H. J. van't.

1860. Comptes rend. 51, 298. “Doubling" by organisms.

1861. "Leçons de chimie. Recherches sur

la dissymétrie moléculaire des produits organiques." 1

${ }^{1}$ Contains theory that atoms in active molecules must be arranged in enantiomorphous form. 
- Pasteur, I.-1884. Rev. scient. de la France et de l'étranger, 33, 2. Molecular dissymmetry.

226. Paterno, E.-1889. Gazz, chim. ital. 19, 195. Hexachlorbenzenes.

22\%. - Pendleton, H. See Michael, A.

228. II Perkin, W. H.-18rr and 1881. J. Chem. Soc. 31, 388; (especially pp. 414, 422); 39,409; (espeally p. 442). Coumaric acid derivatives. Compare Ebert, G.

1888. J. Chem. Soc. 53, 695. Chloriumaric and chlormaleic acids said to be active. 229. ' Perkin, W. H., Jr.-1891. J. Chem. Soc. 59, 798. Hexamethylenedicarboxylic acids.

230. - Petrenko-kritschenko, P. See Melikoff, P.

231. - Piloty, O. See Fischer, E.

232. C Piutti, A.-1886. Comptes rend. 103, 104. Asparagines. 1888. Gazz. Chim. ital. 18, 457, 472, 478. (Ref. Ber. 22, 241, 243, 244). Asparagines. 1891. Ber. 24, 228\%. Remarks on Cramer's papers, "Monoximes of succinic acid."

233. Pleissner, M.-1891. Ann. 262, 1. Camphor series. (Communicated by Beckmann, E.)

234. ॥ Plöchl, J._-1883. Ber. 15, 1946. Chlorcinnamic acids. (Erlenmeyer's laboratory.)

“ see Miller, W. von.

235. C Pribram, R.-188\%. Ber. 20, 1840. Specific rotation of dissolved substances; dilution alters the grouping of the atoms.

1888 and '89. Ber. 21, 2599. Monatsh. Chem. 9, 395, 485. (Ref. Ber. 21, 673, 6r2.)-Ber. 22, 6. Alteration of optical activity by inactive substances.

236. Priebs, B.-1884. Ann. 225, 340. An isonitrostyrol $\mathrm{C}_{6} \mathrm{H}_{5} \cdot \mathrm{HC}=\mathrm{CH} . \mathrm{NO}_{2}$.

237. I Prudhomme, M.-1892. Bull. Soc. Indust. de Mulhouse, p. 72. (Ref. Chem. Centrb]. [4] 4, II, 28. Transformation of isomers containing singly-linked carbon-atoms. 
238. C Przybytek, S.-1884 Ber. 17, 1412. Attempt to double mesotartaric acid.

239. ॥ Pückert, M. -1889. Ann. 250, 240, 250. Unsaturated isomers. (Laboratory of Wislicenus.)

240. C Purdie, T. and Walker, J. Wallace-1892. Chem. Soc. Trans., August. (Ref. Chem. News 66, 33.) "Doubling" of lactic acid.

241. - Rach, C. See Bischoff C. A.

242. L Rammelsberg,C.-1869. Ber. 2, 31. Relations between circular polarisation, crystalline form and molecular constitution.

243. - Ramsay, W. See Williams, K. J.

244. C Raoult, F. M.-188\%. Ztschr. phys. Ohem. 1, 186. Racemic acid dissociated by water.

245. || Redzko, W. G. - 1889. Zhurnal Khim. 21, I, 421. (Ref. Chem. Centrbl. [4] 2, I, 533. Derivatives of stilbene and isostilbene. Tolane chlorides. Compare Blank, A. and Eiloart, A.

246. C Reese, L. -1887 and '88. Ann. 242, 9 : Ber. 21, $27 \%$. Leucinephthalovlic acid.

24\%. - Reimann, K. See Janovski, J. V.

248. I Reimer, C. L.-1881 and '82. Ber. 14, 1802; 15, 2628. Two isomeric diphenyisuccinic acids.

249. L Reusch,_.-1869. Berl. Acad. Ber. p. 530. Optically active arrangement of mica-plates.

250. - Riecke, E. See Meyer, V.

251. - Ritschke, A. See Gattermann, L.

252. - Rohde, G. See Miller, W. von.

253. C Romburgh, P. van.-1886 and '8\%. Recueil trav. chim. 5, $219 ; 6,150$. (Ref. Ber. 20, 468, 375.) Hexyl alcoliol.

254. II Roser, W.-1882. Ber. 15, 234\%. Fumaric, maleic and cumaric acids.

11 " and Haseloff, E.-188\%. Ber. 20, 1576. Dibromcinnamic acids.

255. ॥ Rügheimer, L-1882. Ber. 15, 1625. Diphenylfumaric and maleitc acids. 
256. N Russanow, A.-1891. Ber. 24,349\%. Phenylglyoximes (Hantzsch's laboratory.)

25\%. II Sabanejeff, A.-1875. Ann. 178, 109. Ethylene biniodides.

258. Sachse, H.-188\%. Ber. 21, 2530. Benzene. Compare Wagner, J.

1890. (Ber. 23, 1363.-R. 15.) Hexamethylene.

1892. Ztschr. phys. Chem. 10, 203. Polymethylene rings.

See Liebermann, C.

259. L Sarrau, E.-186\%. Jour. de mathématiques pures et appl. [\%] 1\%, 1. Necessity of optical activity in enantiomorphous structures.

260. \| Saytzeff, Al.-1892, J. prakt. Chem. [2] 45, 300, Oleic and elaidic acids. A confirmation of Wislicenus' views.

261. L Scacchi, - - 1865. Rendiconti dell Acad. di Napoli p. 250. "Doubling" of racemates by crystallisation.

262. C Schardinger, F. 1890. (Monatsh. Chem. 11, 558.R. 6.) Compare Linoissier, G.

263. - Scheibler, C. See Kiliani, H.

264. - Sehiff, R.-1892. Ber. 25, 1690. Peculiar case of isomerism.

265. N Schindler, 'T.-1891. Monatsh. Chem. 12, 63\%. (Ref. Ber. 25, 196.) Crotonaldoxime.

266. II Schmidt, E. $-18 \pi 9$ and 1881. Ber. 12, 252; Ann. 208, 249. Tiglic and angelic acids.

C 1888. Ber. 21, 1829. Hyoscyamine and atropine.

26\%. C Schmoeger, M.-1892. Ber. 25, 1452. Milk-sugars.

268. $\mathrm{N}^{\mathrm{v}}$ Schryver, S. B. -1891 . J. Chem. Soc. Proc. p. 39. (Ref. Chem. News, 63, 174.) Two methyldiethylisoamylammonium jodides.

269. - Schulthess, O, See Michsel, A.

270. | Schultz, G.-1878 and '79. Ber. 11, 216; Ann. 196, 1. Diphenic acid. 
2r1. C Schulze, E., Barbieri, J. and Bosshard, E.-1885. Ztschr. physiol. Chem. 9, 63.

C " and Bosshard, E.-1884. Ber. 17, 1610. Amido-acids.

1885 and '86. Ber. 18, 388: Ztschr. physiol. Chem. 10, 134. Leucine and glutamic acid "doubled."

C " and Nïgeli, E.-1886. Ztschr. physiol. Chem.

11, 201. Phenylamidopropionic acid.

2\%2. - Selden, Ch. C. See Anschütz, R.

2\%3. - Siegfeld, M. See Auwers, K.

2\%. - Silber, P.-188\%. Ber. 20, 2594. Pyrrol derivatives. “ See Ciamician, G.

275. - Simmen, C.-1876. "Untersuchungen über die Constitution der chemischen Elemente und deren Verbindungen bei Annahme regelmüsiger Anordnung im Raume." Basle. (Ref. Jsb. Chem. 18\%6, 4.)

276. II Skraup, Z. H.-1891. (Monatsh. Chem. 12, 10\%, 146. -R. 12, 23.) Compare Tanatar, S.

2r\%. N Smith, A. W.-1892. Ber. 24, 4025. Ketoximes. (Hantzsch's laboratory.)

278. N Sönerbaum, H. G.-1891. Ber. 24, 1215. Dioximidosuccinic acids.-Ibid 1381. w-Isonitrosoacetophenone. (Hantzsch's laboratory.) 1892. Ber. 25, 904. Dioximidopropionic acids.

279. C Sorokin, B.-1888. J. prakt. Chem. [2] 37, 320. Connection between composition and optical activity.

280. C Staedel, W.-18r8. Ber. 11, 1752. Crystallisation of racemates.

281. - Stahel, R. See Fischer, E.

282. - Steche, A. Seé Fischer, E.

283. N Stierlin, R.-1889. Ber. 22, 3\%6. Isomeric dioximes of anisil and paratolil. (V. Meyer's laboratory.) 
284. - Stockmeier, H. See Erlenmeyer, E.

285. A Stohmann, F.-1891. Ber. d. k. sïchs, Ges. der. Wissensch. p. 635. (Ref. Ber. \$5, 496.) Heat of combustion of hexalydrobenzol, etc. A confirmation of Baeyer's strain-theory.

286. II Stolz, F.-1886. Ber, 19, 536. $\beta$-iodacrylic acids ( $p$. 542$.

28\%. C Stone, W. E.-1892. Am. Chem. J. 14, 399. Grapesugar and its isomers. Review.

288. - Strecker, O. See Kekulé, A.

289. - Strutt, C. E. See Swedenborg, E.

290. - Struve, O. See Ladenburg, J.

291. II Sudborough, J. J. -1892. Ber. 25, 223\%. Stilbene series; transformation of isomers. (V. Meyer's laboratory.)

- Svedborg, E. See Swedenborg, E.

292. - Swedenborg, Emmanuel, (or Emanuel), [Svedborg]1721. "Prodromus principiorum rerum naturalium sive novorum tentaminum chymiam et physicam experimentalem geometrice explicandi." Amsterdam : John Osterwyk, $16 \mathrm{mo}$.

172\%. Second edition. J. and A. Strander Amsterdam.

1754. Third edition. Hildburghausen, J. G. Harnisch. For notice of this work see Acta Eruditorum, Feb., 1722, pp. 83-87. 184\%. "Some specimens of a work on the Principles of Chemistry with other treatises. 'Transiated from the Latin by Charles Edward Strutt." London : W. Newbery, 8 ro, pp, xlii +253 , ill. Includes a translation of the work last-mentioned (pp. 1179), also an introduction by the translator (pp. i-xlii.).

293. S Sworn, S. A. - 1889. Phil. Mag. [5] 28, 402, 443. 1890. J. Chem. Soc. 58, 238. 
294. - Szymanski, F. See Deichmüller, A.

295. - Tafel, J. See Fischer, E.

296. II Tanatar, S.-1890. Zhurnal Khim. 22, I, 310. (Ref. Ber. 23, 433.) Conversion of maleïc into fumaric acid. See Skraup, Z. H.

I 1891. Ibid. 23, I, 339. (Ref. Ber. 24,9ro.) Action of water on bromsuccinic acid.

$29 \%$ C Tesmer, H. -1885 . Ber. 18, 2606; especially p. 2608. Saccharin.

298. C Thomsen, J.-1880. Ber. 13, 2168, 2264, 2269. Optical activity of carbohydrates.

1880-'86. Ber. 13, 1388, 1808, 2166; 15,328; 19, 2944. Octahedral benzene formula.

299. N Thomson, J. J., and Threlfall, R.-1886. (Proc. Roy. Soc. 40, 329.-R. 40.)

300. - Threlfall, R. See Thomson, J. J.

301. | Tiemann, F.-1891. Ber. 24, 3169. Hydrobenzoïns. C " and Haarmann, R. -1886. Ber. 19, $125 \%$. Isosaccharic acid.

302. Tilden, W.-1888. J. Chem. Soc. 53, 879. Terpenes and benzene.

303. | Tillmanns, H.-1890. Ann. 258, 8\%. Diphenylsuccinic acid. (Laboratory of Wislicenus.)

304. - Tissot, G. See Michael, A.

305. C Tollens, B. $-1880-' 86$. Ann. 206, 226; 227, 228. Ber.

19, 10\%. Glucoses.

$\begin{array}{lll}- & \because & \text { See Deichmüller, A. } \\ \text { - } & \because & \text { See Günther, A. } \\ \text { - } & \text { - } & \text { See Hermann, P. } \\ & & \text { See Wheeler, H. J. }\end{array}$

306. - Trapesonzjanz, Ch. See Bischoff, C. A.

30\%. C Tutton, A. E.-1891. J. Chem. Soc. 59, 233. Crystalline for'm of calcium glycerate.

308. N Vaubel, W.-1891. "Das Stickstoffatom." Giessen E. Ottmann, pp. 8, ill.-Chem. Ztg. 15, 475. (J. prakt. Chem. [2] 44, 13\%.-R. 48.) Ibid. 572. Correction. ${ }^{1}$

1 The benzene formula published by $\mathrm{V}$. as new is now ascribed to Marsh, "whose paper was not abstracted in the Berichte;" (but see Ref. Ber. 21, 879.) Marsh's paper was, however, nine months later than that of Baeyer. See-R. 14 and 48. 

309, - Voit, E. See Bischoff, C. A.

310. ^ Wachter, V.-1890. (Chem. Centrbl. [4] 2, I, 45\%, $505,561,61 \%, 849$. Benzene. A Review. -12. 14.)

311. - Waechter, F.-18\%8. Chem. Centrbl. [3] 9, 648, 66\%. Relative volumes of the atoms.

312. C Wagner, G.-1886 and 's9. Gazz. chim. ital. 16, 390; Ber. 2:, 305\%. Piperylene bromides.

313. W Wagner, J.-1888.-Chem. Centrbl. [3] 19, II, 1333. On the benzene configuration of Sachse.

314. II Walden, P.-1890. Ber. 23, 1958. Stilbene group.

1891. (Ztschr. phys. Chem. 8, 433.-R. 30.) $\because \quad$ See Bischoff, C. A.

315. | Walker, James.-1892. J. Chem. Soc. 61, 696, 1088. Dissociation constants of organic acids.

316. - Walker, J. Wallace. See Purdie, T.

317. C Wallach, M.-1884. Ann. 226, 80. A possible third hydrobenzoïn.

1884-'92. Ann. 225, 314; 227, 27r; 230, $225 ; \mathbf{2 3 8}, 78 ; \mathbf{2 3 9}, 1 ; \mathbf{2 4 1}, 315, \mathbf{2 4 5}$, 191,$241 ; \mathbf{2 4 6}, 221 ; \mathbf{2 5 2}, 34,106,136$;

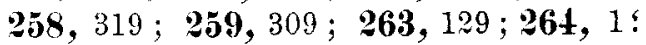
$\mathbf{2 6 8}, 19 \% ; \mathbf{2 6 9}, 326 ; \mathbf{2 7 0}, 171$. Terpenes. 1891. Ber. 24, 1525. Terpenes; review.

C $\because$ and Brass, W.-1884. Ann. 225, 291. Terpenes.

C $\quad$ and Conrady, C. $-1889 . \quad$ Ann. 252, 141. Terpenes.

C $\because \quad$ and Gildermeister, E.-1888. Ann. 246, 265. Terpenes.

C $\lll$ and Otto, A.-1889. Ann. 25.3, 149. Terpenes.

318. - Warder, R. B.-1890. Am. Ass. Adv. Sci. 39, 111. Stereochemistry. Review with bibliography. 
319. - Warrington, A. See Meyer, V.

320. C Weger, F.-1883. Ann 221, 68. Styrolene is a mixture. Compare Berthelot, D.

321. N Wegerhoff, P.-1889. Ann. 252,11. Methylbenzophenone oxime. Communicated by Beckmann.

322. Wendt, G.-1890. Deutsche Chem. Ztg. 5, 194. (Ref. Chem. Centrbl. [4] 2, II, 196.) Benzene and naphthalene.

323. - Wenzel, C. F. -1r\%r. "Lebre von der Verwandschaft der Körper." Dresden, G. A. Gerlach, $16 \mathrm{mo}, \mathrm{pp}$. iii +492 . Affinity is explained by the forms of the smallest particles. See especially pp. 8 , et seq.

324. N Werner, A.-1890. "Ueber rüumliche Anordnung der Atome in Stickstoffhaltigen Molekülen." -Ber. 23, 2331, 2333, 2336. Oximes.

1891. Züricher Vierteljahrsschrift 36 (Ref. Ztschr. phys. Chem. 9, 220). "Beiträge zur Theorie der Affinität und Valenz."

1892. (Ber. 25, 2\%.-R. 45.) Hydroxamic acid derivatives.

" See Hantzsch, A.

325. - West, - -1873. Bull. Soc. Chim. (Paris), [2] 19, 544. On "L'architecture du monde des atomes" of Gaudin.

326. C Wheeler, H. J. and Tollens, B.-1889. Ann. 254, 314. Xylose isomeric with arabinose.

32\%. - Wiegand, E.-See Beilstein, F.

328. C Will, W.-1885. Ber. 18, 1311. Sorbite, isodulcite, elc. ; especially p. 1321.

188\%. Ber. 20, 1189 . Sugar's.

1888. Ber. 21, 1\%1\%. Atropine and hyoscyamine.

1891. Ber. 24, 400. Oxypyroracemic acid. $\mathrm{CO}_{2} \mathrm{H} . \mathrm{CO} . \mathrm{CH}_{2} \mathrm{OH}$.

C $"$ and Bredig, G.-1888. Ber. 21, 277\%. Hyoscyamine and atropine. 
329. N Willgerodt, C. -1888 and 1890. (J. prakt. Chem. [2] 3\%, 449: 41, 291, 526; 42,63.-R. 39.) 1892. Verein deutscher Naturforsch: (i3,

II. 66. Elements of the nitrogen group. --J. prakt. Chem. [2] 45, 5it. J)initrosoazobenzene: in answer to Kehrmann and Nessinger.-Ibia. 46, 128 . Nitrosoazo computuds.

$\mathrm{N}$ “ and Herman, B.-1890. J. prakt. Chem. $[\because] 4 \%, 12 \%$

330. N Williams, K. J., and Ramsay, W.-1886. Proc. Chem. Soc., p. 223. Supposed existence of an allotrupic modification of nitrogen: compare Johnson, G. S.

331. - Wing, J. F. See Michael, A.

332. II Wislicenus, J.-18\%3. (Ann. 167, 302.-R. 2). Lactic acids: (especia ly pp. 343-345).

188\% (bh. kgl. Süchs. Ges. der Wissensch.

14, 1. "Ueber die riumliche Anordnung der Atome in organischen Molekülen und ihre Bestimmung in geometrisch-isomeren ungesättigten Verbindungen." Leipzig. Hirzel.

188\%. Verein deutsch. Naturforscher, Tageblatt 240. Addition of bromine to acetylenedicarboxlic acid. (Compare Bandrowski, also Michael, A.)-Ber. $\mathbf{2 0}$, 1008. Derivatives of crotonic acids. Two monochlorpropylenes.

1888. (Ber. 21, 581.-R.11.) Answer to Lossen's paper in Ber. 20, p. 3306.-Ann. 246, 53. Fumaric and maleîc acids.-Ann. 248, 281. Crotonic acid and derivatives. Reply to Michael, A.

1889. Second, unaltered, edition of "Rüumliche Anordnung;" with notes, pp. 79, Svo.-Verein Deutsch. Natur- 
Wislicenus, J.-forsch. 19 Sept. Atomic arrangement.(Ann. 250, 224. -R. 23.) Derivatives of pseudo-butylene, angelic and tiglic acids. 1890. Ueber die Umwandlung stereoisomerer ungesättigter Verbindunger ineinander bei höherer Temperatur. Leipzig, pp. $32,4^{\circ}$.

$\begin{array}{lll}- & \because & \text { See Blank, A. } \\ - & \because & \text { See Eiloart, A. } \\ - & \because & \text { See Hoff, J. H. van't. }\end{array}$

- " " See Hölz, O.

- " $\quad$ See Just, F.

$" \quad$ See König, Arn.

“ See 'Tillmanns, H.

333. - Wunderlich, Ae.-1886. " Configuration organischer Moleküle." Würzburg.-R. 11), (Ref. Ber. 19, 592.)

334. C Wolff, I.-1885. Ann. 229, 249. Two lactones of levulic acid.

335. L Wurtz, Ad.-185\%. Ann. chim. phys. [3] 51, 358. Caproic acid.

336. - Wurtz, Henry. - 1876. Am. Chemist 6, 321, 440. Geometrical chemistry. Volumes of Molecules.

337. C Wyrouboff, G.-1884-'86. Bull. Soc. chim. (Paris), [2] 41, 210; 45, 52; Comptes rend. 102, 62. Crystallisation of racemates and tartrates.

1886. Ann. chim. phys. [6] 8, 340. Reusch's active piles.-Ibid. 9, 221 . "Doubling" racemates.

338. I Zelinsky, N.-1888. Zhurnal Khim. 20, 672. (Ref. Chem. Centrbl. [4] 1, I, 782.) Dimethylsuccinic acids.

1889. Ber. 22, 2823. Dimethylglntaric acids.

1891. Ber. 24, 3997. Uimethyladipic acids. Ber. 24, 4006. Dimethyloxyglutaric acids. 
I Zelinsky, N., and Besredka, A.-1891. Ber. 24, 459. Stereomeric trimethylsuccinic acids. (Compare Bischoff.)

1 " and Buchstab. L.-1891. Вей. 24, 18:6. Phenylmethylsuceinic acids.

$1 \quad "$ and Krapivin, S.-1889. Ber. 92, 646. Dimethylsuceinic acids.

339. I Zincke, Th.-18\% and ':9. Ber. 10, 999; Ann. 19s, 198. Hydrobenzoins.

1884. Ber. 1i, ios. Phenylmethylglycols. $\mathrm{N}^{\mathrm{v}}$ 1890. Ber. 23, 1315. Azo derivatives of phenyl- $\beta$-naphthylamine; especially $\mathrm{p}$. 1321.

340. - Zuurdeeg, J. See Klinger, H.

CHRONOLOGICAL CLASSIFICATION OF PUBLICATIONS.

The numbers refer to the preceding list. Those occurring in more than one perjod are printed in heary type.

First Period. Before the year 1800.

292, 323.

Second Period. 1800 to $18 \% 3$ inclusive.

12, 26, 29, 33, 47, 48, 51, 58, 63, 89, 94, 95, 122, 135, 140, 146, 162, 166, 168, 180, 209, 223, 225, $335,242,249,250,261$, $325,332,335$.

Third Period. 18:4 to $1884^{*}$ inclusive.

$13,15, \mathbf{1 7}, 21,23,35, \mathbf{3 8}, \mathbf{4 0}, 45,50,51,69, \mathbf{8 1}, \mathbf{8 3}, 90$, 107, 118, 120, 121, 127, 133, 138, 140, 141, 143, 146, 15\%, 159, $162,163,164,166,168,170,174,179,184,191,199, \mathbf{2 0 0}, \mathbf{2 0 4}$, 205, 209, 211, 225, 228, 234, 236, 238, 248, 254, 255, 25\%, 266, $270,271,275,280, \mathbf{2 9 8}, 305,311,317,320,325,336, \mathbf{3 3 7}, \mathbf{3 3 9}$.

\footnotetext{
*Von Baeyer began to develop Van't Hoff's theory in 1885, Wislicenus' in 1886 .
} 
Fourth Period. $1885^{*}$ to 1892 inclusive.

$1,2,3,4,5,6,8,9,10,11,12,14,17,18,20,25,27,28,31$, $\mathbf{3 3}, 34, \mathbf{3 8}, 39, \mathbf{4 0}, 41,43,46,49, \mathbf{5 1}, 53,54,57,59,60,65,66$, $68,70,71,73,74,75,76,78,79,80,81,82,83,84,85,87,88,92$, $93,97,98,99,100,101,102,103,104,105,106,107,108,109$, $111,112,113,115,116,119,122,123,125,126,127,128,129$, $131,132,13 \%, 138,142,144,145,148,151,152,154,156,158,161$, 166, 168, 169, 170,173, 174, 175, 176, 178, 179, 133, 185, 188, 189, 190, 193, 196, 198, 200, 201, 202, 203, 204, 20\%, 210, 214, $215,217,218,219,220,221,222,223,226,228,229,232,233$, $235,237,239,240,244,245,246,253, \mathbf{2 5 4}, 256,258,260,262,264$, 265, 266, 267, 268, 271, 2r4, 276, 27\%, 278, 279, 283, 285, 286, $287,291,293,296,297,298,299,301,302,303,305,307,308$, $310,312,313,314,315,31 \%, 318,321,322,324,326,328,329$, 330, 332, 333, 334, 337, 338, 339.

\section{LIST OF REVIEWS.}

\section{STEREOCHEMISTRY.}

Auwers, K., 1890. “Entwicklung der Stereochemie," pp. $15 \%$. Hoff, J. H. van't. "Dix Années" and its translations; see index. Especially valuable is the latest edition (Vau't HoffMeyerhoffer, 1892) which is furnished with author- and subject-index.

Meyer, Victor, 1890. Ber. 23, 567-619. "Ergebnisse und Ziele Stereochemischen Forschung."

Warder, W. B., 1890. Am. Ass. Adv. Sci., 39, 111-129; with der Bibliography, 130-136.

\section{Benzene and Derivatives.}

Noyes, W. A., 1889. Am. Chem. J., 11, 48\%-496.

Wachter, V., 1890. Chem. Centrbl. [4] 2, I, 457, 505, 561, 617, 849.

Wallach, M., 1891. Ber. 24, 1525, 1579. Terpenes.

Hydroxylamine and Derivatives.

Keiser, E. H., 1889. Am. Chem. J., 11, 480-487. 


\section{Sugars.}

Fischer, E., 1890. Ber. 23, 2114. Syntheses; with Bibliography.

Keiser, E. H., 1889-'90. Am. Chem. J., 11, 2\%\%, 12, 35\%, pp. 19. Stone, W. E., 1892. Am. Chem. J., 14, 399-405. Configuration of grape-sugar and its isomérs.

\section{TAUTOMERISM.}

The literature of this subject has no place in the index, as its bearing on stereochemistry is too indirect, and it seems sufficient to refer here to the review by

Orndorff, W. R., 1892. Am. Chem. J., 14, 238-246.

Note on the Stereochemistry of Creatinines.

These compounds seem to represent a new class of isomers. According to Johnson there are, including Liebig's base, four distinct substances having the composition of creatinine (besides three which differ in composition from these in that each contains two molecules of water). The fact that no more than one creatine has yet been obtained from the four creatinines shows they are probably identical in structure. In every case, so far as is known,<smiles></smiles>

Now corresponding to this structural formula for creatinine there are, according to the Hantzsch-Werner theory, two stereomers :

I.

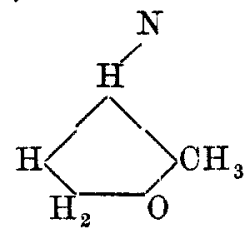

and II.,

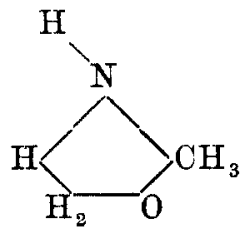


but if the nitrogen valences maintain in this compound what is supposed to be their normal inclination of $120^{\circ}$ (compare Bischoff, Ber. 23, 1974), then the methyl-group and the corresponding hydrogen-atom are not in the plane of the ring, and each of the above formulae represents two compounds in one, of which $\mathrm{H}$ and $\mathrm{CH}_{3}$ are on the same side, in the other on opposite sides of the plane of the ring. I. represents :
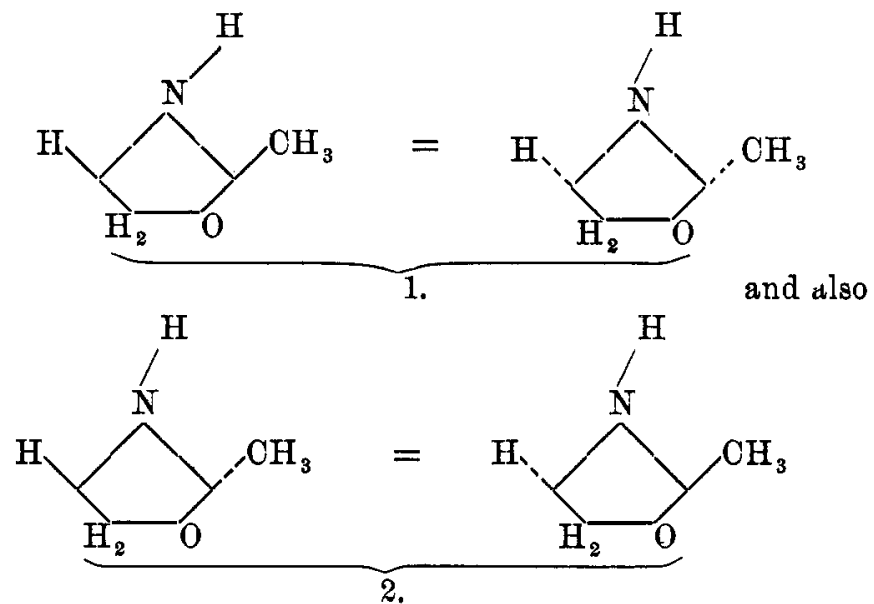

II. represents :

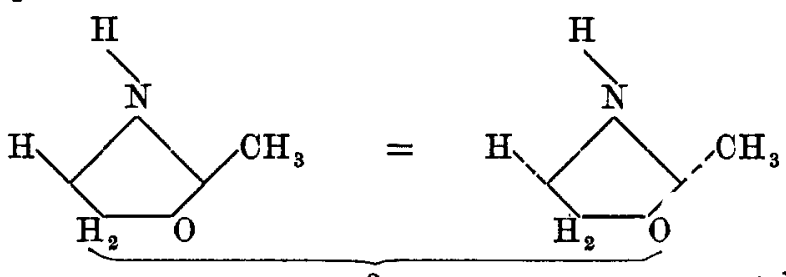

3. and also

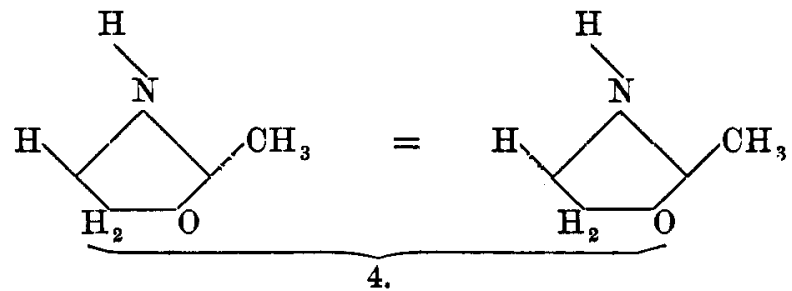


Theoretically, then, as practically, four creatinines are found to exist; and if this theory proves correct we must add to the nitrogen stereomers foreseen by Hantzsch and Werner another class cousisting of the nitrogen analogues of the cis-trans isomers of Van't Hoff and von Baeyer. Of this class the creatinines would be the first known representatives.

Chemical Laboratury.

Now York Post-Graduate Medical Sehool. 